\title{
AVALIAÇÃO DA SELEÇÃO DE FORNECEDORES DE CERÂMICA VERMELHA COMO PRÁTICA AMBIENTAL NA CADEIA DE SUPRIMENTOS VERDE DE CONSTRUTORAS
}

\author{
F. H. F. DE MEDEIROS*, H. C. D. PIMENTA \\ Instituto Federal de Educação, Ciência e Tecnologia do Rio Grande do Norte \\ fernando@verdesolucoes.com.br*
}

Submetido 25/11/2019 - Aceito 30/07/2020

DOI: $10.15628 /$ holos.2020.9198

\section{RESUMO}

O presente estudo objetiva avaliar os critérios de seleção de fornecedores de cerâmica vermelha como práticas ambientais adotadas no gerenciamento da cadeia de suprimentos verde de construtoras que atuaram em meados de 2012 no setor imobiliário de Natal-RN. Para tanto, foi desenvolvido uma survey nas construtoras credenciadas ao Sindicato da Construção Civil do Rio Grande do Norte. Como metodologia, utilizou-se um questionário com um grupo de 23 variáveis, abordando os critérios de seleção de fornecedores de cerâmica vermelha. Diante dos critérios de seleção de fornecedores, $35,7 \%$ das construtoras apontaram que sempre adotam o preço do produto como critério e $21,4 \%$ preferem as licenças ambientais. De outro modo, apenas $7,1 \%$ das construtoras exigem uma postura mais proativa de seus fornecedores a partir da implementação da ISO 14001. Frente aos resultados, a pesquisa conclui que as empresas de construção civil estudadas possuem uma postura preventiva e não realizam sistematicamente práticas da CSV, comprometendo, assim, o desempenho ambiental da montante à jusante.

PALAVRAS-CHAVE: cadeia de suprimentos verde, critérios de seleção de fornecedores, construção civil, cerâmica vermelha.

\section{EVALUATION OF SELECTION OF RED CERAMIC SUPPLIERS AS AN ENVIRONMENTAL PRACTICE IN THE GREEN SUPPLY CHAIN OF CONSTRUCTING COMPANIES}

\begin{abstract}
This study aims to evaluate the selection criteria of red ceramic suppliers as environmental practices adopted in the green supply chain management of construction companies that worked in mid-2012 in the Natal-RN real estate sector. For this, a survey was developed in the construction companies accredited to the Union of Civil Construction of Rio Grande do Norte. As a methodology, we used a questionnaire with a group of 23 variables, addressing the selection criteria of red ceramic suppliers. Given the selection criteria of suppliers, $35.7 \%$ of builders
\end{abstract}

pointed out that always adopt the price of the product as a criterion and $21.4 \%$ prefer environmental licenses. Otherwise, only $7.1 \%$ of construction companies require a more proactive stance from their suppliers from the implementation of ISO 14001. Based on the results, the survey concludes that the construction companies studied have a preventive stance and do not systematically practice CSV, thus compromising the environmental performance of the upstream downstream.

KEYWORDS: green supply chain, supplier selection criteria, building construction, red ceramics. 


\section{INTRODUÇÃO}

Frente aos impasses dos impactos ambientais decorrentes dos setores da construção civil, é importante frisar as práticas ambientais dentro do contexto de todo o processo produtivo. Sob esse viés, Jabbour e Jabbour (2009) consideram que, na gestão ambiental empresarial, para serem alavancadas expectativas e metas através da mitigação dos efeitos negativos por atividades empresariais, é necessário construir um conjunto de adaptações no segmento organizacional, a fim de enfrentar a complexidade de inserir a variável ambiental.

Nesse sentido, por meio do planejamento estratégico, é possível definir como a empresa irá interagir com seus fornecedores na gestão do relacionamento com fornecedores (Lambert, 2008). Esse tipo de gestão aborda critérios competitivos tendendo à aquisição de produtos e serviços de menor criticidade, além dos cooperativos e direcionados para fornecedores-chave (Bozarth \& Handfield, 2008; Lambert, 2008).

Segundo Lambert (2008) e Bozarth e Handfield (2008), os critérios de escolha e de avaliação de fornecedores não incluem somente medidas quantitativas (taxas de entrega, entre outros), mas também fatores qualitativos, tais como estabilidade do gerenciamento, confiabilidade e cumprimento de regulamentações ambientais. Consequentemente, a natureza do problema de seleção de fornecedores envolve múltiplos critérios, o que torna necessária a inclusão de abordagens para agregá-los adequadamente (Ehrlich, 1996).

Diante da necessidade de conformidade em relação à legislação ambiental surgida na atualidade, a Gestão da Cadeia de Suprimentos Verdes (GCSV) aparece como uma estratégia fundamental para uma maior integralização de práticas ambientais que venham a expandir as melhorias da cadeia produtiva, no que concerne ao seu desempenho proativo. Sendo assim, a GCSV, no desenvolvimento sustentável, é definida pela cooperação, visando à gestão de informações e aos fluxos de capital ao longo de toda cadeia. Nesse contexto, vários atores são envolvidos, a saber, os fornecedores externos, as funções internas das empresas, os distribuidores externos, os clientes comercias e os usuários finais (Hervani, Helms \& Sarkis, 2005; Seuring \& Müller, 2008).

Na GCSV, podem ser verificadas desde ações reativas, a partir do monitoramento de programas ambientais adotados por fornecedores, até a adoção de práticas proativas, incluindo colaboração com fornecedores, práticas externas da GCSV, recuperação dos investimentos e ecodesign (Zhu \& Sarkis, 2004). Desse modo, existe uma carência de estudos no que concerne à seleção de fornecedores de cerâmica vermelha adotadas como prática ambiental pelas construtoras da Grande Natal. A partir dessa problemática, surgiu a seguinte indagação: como as construtoras associadas ao SINDUSCON-RN estavam trabalhando a variável ambiental no tocante aos critérios de seleção de fornecedores de cerâmica vermelha?

Sendo assim, o presente estudo teve por finalidade avaliar os critérios de seleção de fornecedores de cerâmica vermelha como práticas ambientais adotadas no gerenciamento da cadeia de suprimentos verde, usada por construtoras que atuaram em meados de 2012 no setor imobiliário de Natal-RN. Para isso, buscou-se analisar os critérios competitivos e ambientais da seleção de fornecedores de cerâmica vermelha, especificamente, telhas e tijolos.

Portanto, no referencial teórico do presente trabalho, é abordada a temática referente à Cadeia de Suprimentos Verdes (CSV). Em seguida, na metodologia, apresenta-se a pesquisa relacionada a questões de natureza qualitativa, com uso do método survey e do questionário para 
a coleta de dados, com um 01 grupo de 23 variáveis. Por fim, foi feita a compilação e a análise dos dados obtidos.

\section{CADEIA DE SUPRIMENTOS VERDES}

No que concerne à CSV, pode-se dizer que ela se define por interações desenvolvidas entre os fornecedores e a empresa foco. Nesse segmento, a organização em questão necessita dar menos ênfase aos amplos benefícios econômicos em detrimento de um melhor desempenho ambiental e social, haja vista que ela é o eixo central da Cadeia de Suprimentos Verde. Sendo assim, surge a necessidade de coletar informações sobre cada nível de produção, fazendo uso de resultados ambientais e sociais, além de garantir a participação na melhoria do desempenho dos principais fornecedores (Seuring \& Müller, 2008).

Por outro lado, Hervani, Helms e Sarkis (2005) afirmam que a GCSV exerce uma forte influência na redução dos impactos ambientais de uma empresa com uma proposta de partilha de responsabilidades interorganizacionais, a serem apoiadas pelas ferramentas de gestão ambiental e, assim, mapeadas ao longo da CSV para diagnosticar o desempenho ambiental. Com essa abordagem, as pressões internas, como o monitoramento, avaliam a carga ambiental associada à prestação de serviços e, consequentemente, às suas práticas, sejam elas reativas, preventivas ou proativas.

Em linhas gerais, a conscientização das empresas voltadas aos ganhos no contexto ambiental é resultante de uma cultura disseminada dentro das próprias organizações. Em consequência, o amadurecimento das suas práticas de gestão ambiental é atribuído pelo aumento das pressões dos regulamentos e pelo intenso mercado interno, sendo eles os motivadores da implementação da GCSV. No entanto, isso não significa que haverá melhorias no rendimento econômico (Zhu, Sarkis \& Lai, 2007a). Nesse sentido, as fontes de diminuição de custos podem ser identificadas pela otimização de recursos ambientais de forma eficiente e pela redução das perdas no processo produtivo, cabendo às empresas só adotarem práticas da CSV quando verificarem benefícios concernentes aos resultados financeiros e operacionais (Brito \& Berardi, 2010).

Desse modo, as empresas devem ir além dos requisitos das leis e regulamentos, promovendo conscientização interna, essencial para a implementação de práticas ambientais, uma vez que eles são motivadores e condutores para o seguimento da GCSV (Zhu, Sarkis \& Lai, 2007b). Sendo assim, mediante o amadurecimento do nível organizacional das empresas, os critérios de seleção de fornecedores aparecem como uma oportunidade de aumentar os níveis de desempenho ambiental dentro da CSV (Jabbour \& Jabbour, 2009). Diante disso, tais critérios são instituídos no processo de compra pela empresa foco, a qual exige que seus fornecedores apresentem requisitos, como, por exemplo, a ISO 14001 (Jabbou \& Jabbour, 2009; Nawrocka, Brorson \& Lindhqvist, 2009), que é fundamental para corroborar com o "greening" da cadeia de suprimentos, tendo em vista que reduz os impactos ambientais, aumentando o desempenho ambiental da CSV. 


\section{METODOLOGIA}

A pesquisa trata-se de uma survey, uma vez que possibilita, ao avaliar o fenômeno a ser estudado, conhecer a realidade do cenário sem controlar as variáveis dependentes e independentes (Freitas, Oliveira, Saccol \& Moscarola, 2000). Segundo Günther (2003), a survey sustenta-se por obter a coleta de dados por meio do questionário, buscando identificar características inerentes aos objetivos traçados pela pesquisa de acordo com o objeto de estudo.

Além disso, o presente estudo foi desenvolvido com a abordagem quantitativa dos dados, já que teve o objetivo de assegurar uma generalização dos resultados com a possibilidade de controlar a situação dentro do contexto, de modo a criar ambientes artificiais. Vale destacar que essa abordagem retira a possibilidade de interferência entre as variáveis e aumenta o grau de intersubjetividade entre o pesquisador e o objeto de estudo que utiliza esse tipo de procedimento (Günther, 2006).

\subsection{Procedimentos de campo}

A revisão de literatura foi realizada, inicialmente, pela seleção dos artigos para o embasamento teórico, a fim de que fosse possível haver a compreensão do tema. Essa triagem foi empregada em 2012, principalmente, em bases de pesquisas internacionais (Scienc Direct e Scopus) e nacional (Scielo), além de revistas e, em casos excepcionais, livros e estudos de instituições (sites). Para a delimitação da revisão de literatura, foram utilizadas palavras-chaves como "Green Supply Chain" e "Green Buildings".

Diante disso, procurou-se, por meio de um alinhamento, fazer uma triagem a partir da relevância dos artigos. Esse procedimento, por sua vez, consistiu em quatro passos, sendo eles: a leitura do título, a leitura do resumo, a leitura das considerações finais e a verificação, a partir da ferramenta disposta no Google Scholar, da quantidade de citações dos artigos. Posteriormente, os trabalhos que, nessas etapas, atenderam ao nosso interesse foram lidos na íntegra e, assim, foram realizados fichamentos. Para o desenvolvimento do questionário, foi investigado, prioritariamente, estudos empíricos nos quais as metodologias pudessem satisfazer os objetivos e discussões pertinentes, para que fosse viável confrontá-los com os resultados compilados perante a indagação construída.

\subsection{Descrição do instrumento de coleta de dados}

$\mathrm{O}$ instrumento de coleta de dados adotado no presente trabalho foi um questionário estruturado com um grupo de 23 variáveis, dividido em variáveis competitivas e ambientais, como critérios de seleção de fornecedores de cerâmica vermelha, uma vez que, de acordo com Freitas et al. (2000), questionários estruturados são ideais para coletar os dados em surveys. Além disso, foi estabelecido o uso da escala tipo ordinal, Likert, com seis alternativas, contemplando os graus de opinião (0-6) e a qualificação das respostas possíveis, que se organizam em grupos para cada questionamento aplicado às definições requeridas (Günther, 2003).

O grupo de variáveis teve por finalidade analisar os critérios competitivos e ambientais estabelecidos pelas construtoras nos processos de seleção de fornecedores de cerâmica vermelha. Nesse contexto, Jabbour e Jabbour (2009) explicam que a inserção das variáveis ambientais nas 
empresas vem sendo adotada gradativamente como critérios de seleção de fornecedores, contribuindo para os fatores que aumentam as possibilidades de selecioná-los com alto desempenho ambiental, resultando, assim, no amadurecimento do nível de gestão ambiental devido à disponibilidade de informações desejadas.

Nessa perspectiva, Nawrocka, Brorson e Lindhqvist (2009) afirmam que a certificação da ISO 14001 pode ser um facilitador nas relações entre cliente e fornecedor, passando a criar uma melhor comunicação de confiança no que diz respeito aos requisitos ambientais, assim como os critérios de competitividade (Souza, Kliemann Neto \& Anzanello, 2012).

Portanto, a colaboração ambiental realizada a partir dos critérios de seleção das construtoras com os seus fornecedores é uma importante estratégia para reduzir os impactos ambientais, podendo ser verificado um relacionamento de compras "amigáveis" entre compradores e fornecedores quando cumpridas as exigências entre as partes (Azevedo, Carvalho \& Machado, 2011).

Dessa forma, esse grupo de variáveis foi descrito pela sigla "GA_CSFM" (utilização de critérios de seleção de fornecedores de cerâmica vermelha - da variável "GA_CSFM.1" a variável "GA_CSFM.23"). Nessa perspectiva, para a chave de resposta deste grupo, foi dada a seguinte escala, que varia de acordo com a implementação dos critérios nas obras: nunca ( $0 \%$ do material é fornecido - critério adotado para seleção do fornecedor); quase nunca (até $20 \%$ do material é fornecido); às vezes (de 20 a $50 \%$ do material é fornecido); quase sempre (50 a $80 \%$ do material é fornecido); sempre (de 80 a 100\% do material é fornecido); sem opinião (não se aplica ou não quer opinar). Posto isso, as variáveis estão descritas no Quadro 1 a seguir.

Quadro 1: Grupo de Critérios de Seleção de Fornecedores.

\begin{tabular}{|c|c|c|}
\hline \multicolumn{2}{|r|}{ Variáveis } & \multirow{2}{*}{$\begin{array}{c}\text { Base teórica para } \\
\text { definição da das } \\
\text { variáveis }\end{array}$} \\
\hline Código & Descrição & \\
\hline GA_CSFM.1 & Preço do produto & \multirow{18}{*}{$\begin{array}{c}\text { (Jabbour \& Jabbour, } \\
\text { 2009; } \\
\text { Nawrocka, Brorson } \\
\text { \& Lindhqvist, 2009; } \\
\text { Souza, Kliemann } \\
\text { Neto \& Anzanello, } \\
\text { 2012; } \\
\text { Azevedo, Carvalho \& } \\
\text { Machado, 2011) }\end{array}$} \\
\hline GA_CSFM.1 & Proporcionar facilidade na compra (formas pagamento) & \\
\hline GA CSFM. 2 & Qualidade dos materiais & \\
\hline GA_CSFM.3 & Confiabilidade no prazo de entrega & \\
\hline GA_CSFM.4 & Possuir as licenças ambientais em dia & \\
\hline GA_CSFM.5 & Possuir a ISO 14001 implementada & \\
\hline GA_CSFM.6 & Possuir a ISO 14001 implementada e certificada & \\
\hline GA_CSFM.7 & Possuir a Certificação OHSAS 18001 & \\
\hline GA_CSFM.8 & Possuir a BS 8800 & \\
\hline GA_CSFM.9 & $\begin{array}{l}\text { Firmar cláusulas contratuais que estabelecem as rotinas } \\
\text { operacionais para o controle ambiental }\end{array}$ & \\
\hline GA_CSFM.10 & Permitir visitas técnicas no processo produtivo & \\
\hline GA_CSFM.12 & Realizar auditorias ambientais periodicamente & \\
\hline GA_CSFM.13 & Apresentar relatórios de ações ambientais e sociais & \\
\hline GA_CSFM.14 & Possuir programa de logística reversa & \\
\hline GA_CSFM.15 & Possuir um Plano de Gerenciamento de Resíduos Sólidos & \\
\hline GA_CSFM.16 & Possuir uma gestão de resíduos perigosos & \\
\hline GA_CSFM.17 & Possuir práticas de reciclagem interna & \\
\hline GA_CSFM.18 & Possuir práticas de reciclagem externa & \\
\hline
\end{tabular}




\begin{tabular}{|l|l|}
\hline GA_CSFM.19 & $\begin{array}{l}\text { Possuir um plano de monitoramento e controle das } \\
\text { emissões atmosféricas }\end{array}$ \\
\hline GA_CSFM.20 & Possuir programa de produção mais limpa \\
\hline GA_CSFM.21 & Utilizar a metodologia de análise do ciclo de vida \\
\hline GA_CSFM.22 & Possuir programas de rótulos ambientais \\
\hline GA_CSFM.23 & Listar as substâncias perigosas usadas no processo \\
\hline
\end{tabular}

\subsection{Procedimento de amostragem, coleta de dados e tabulação}

Com relação à amostragem da pesquisa, foi firmada uma parceria com o Sindicato da Indústria da Construção Civil do Rio Grande do Norte (SINDUSCON-RN). Desse modo, o SINDUSCON-RN apresentou uma listagem de 65 empresas que operavam na Grande Natal no setor imobiliário. Assim, foi estabelecido inicialmente contato telefônico com as construtoras e, logo em seguida, foi realizada a aplicação dos questionários-teste. Posteriormente, foram enviados os questionários para as 65 empresas, em novembro de 2012, estabelecendo um prazo de 15 dias para o retorno, período que foi prorrogado por mais 15 dias.

Diante dos questionários respondidos, tabulou-se os dados para melhor agrupamento das variáveis e geração dos gráficos, visando à maior facilitação do entendimento dos resultados.

\section{RESULTADOS E DISCUSSÃO}

A Figura 1 apresenta um gráfico com os resultados dos critérios e as práticas adotadas na seleção de fornecedores de materiais de cerâmica vermelha, utilizados para avaliar o interesse dos fornecedores em aprimorar o seu desempenho pelo uso do envolvimento direto da construtora. Sendo assim, para reforçar os resultados obtidos nessa figura, a Figura 2 traz o coeficiente de variação dos critérios de seleção de fornecedores, medindo a dispersão em torno da média aritmética dos resultados.

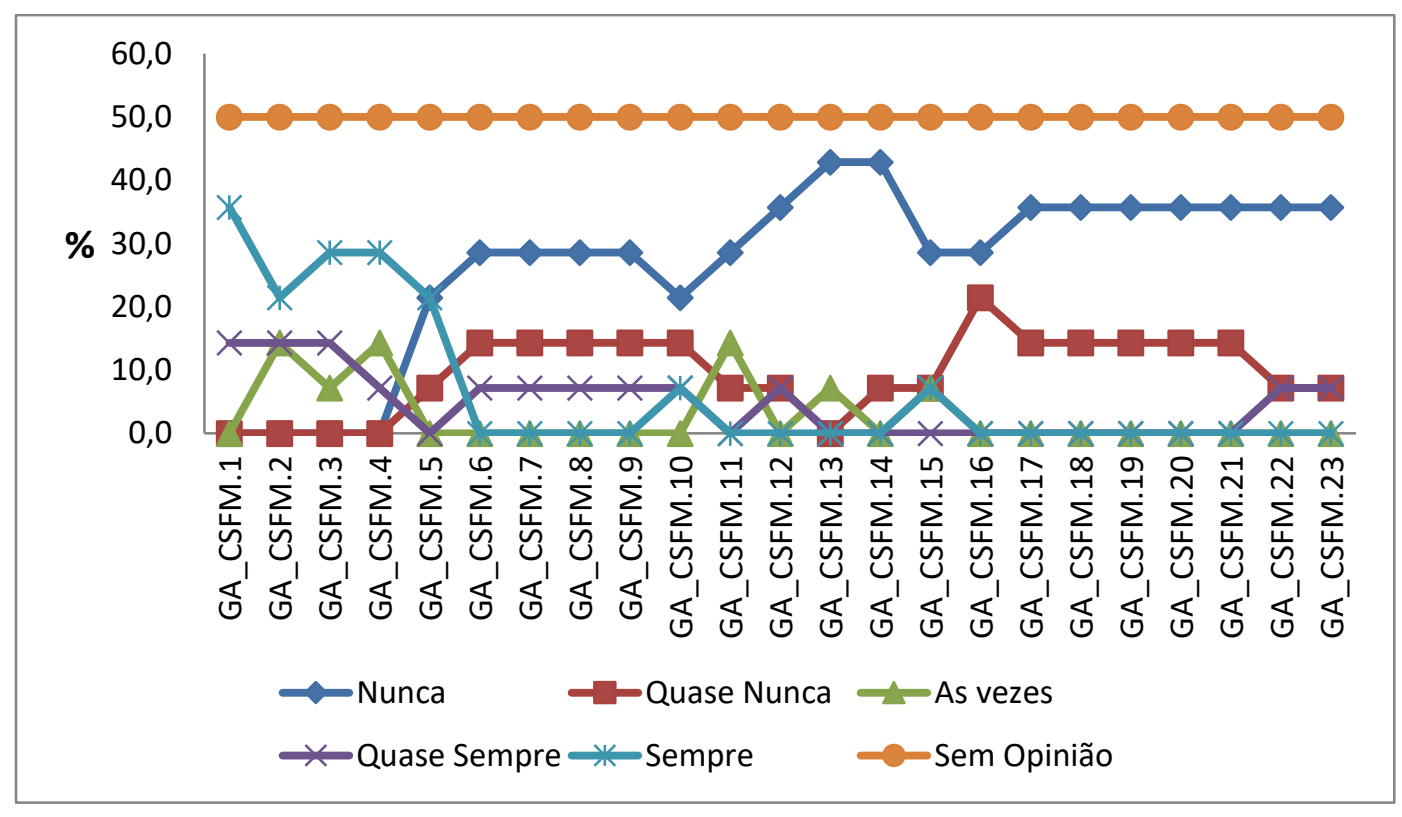

Figura 1: Adoção dos critérios de seleção de fornecedores. 


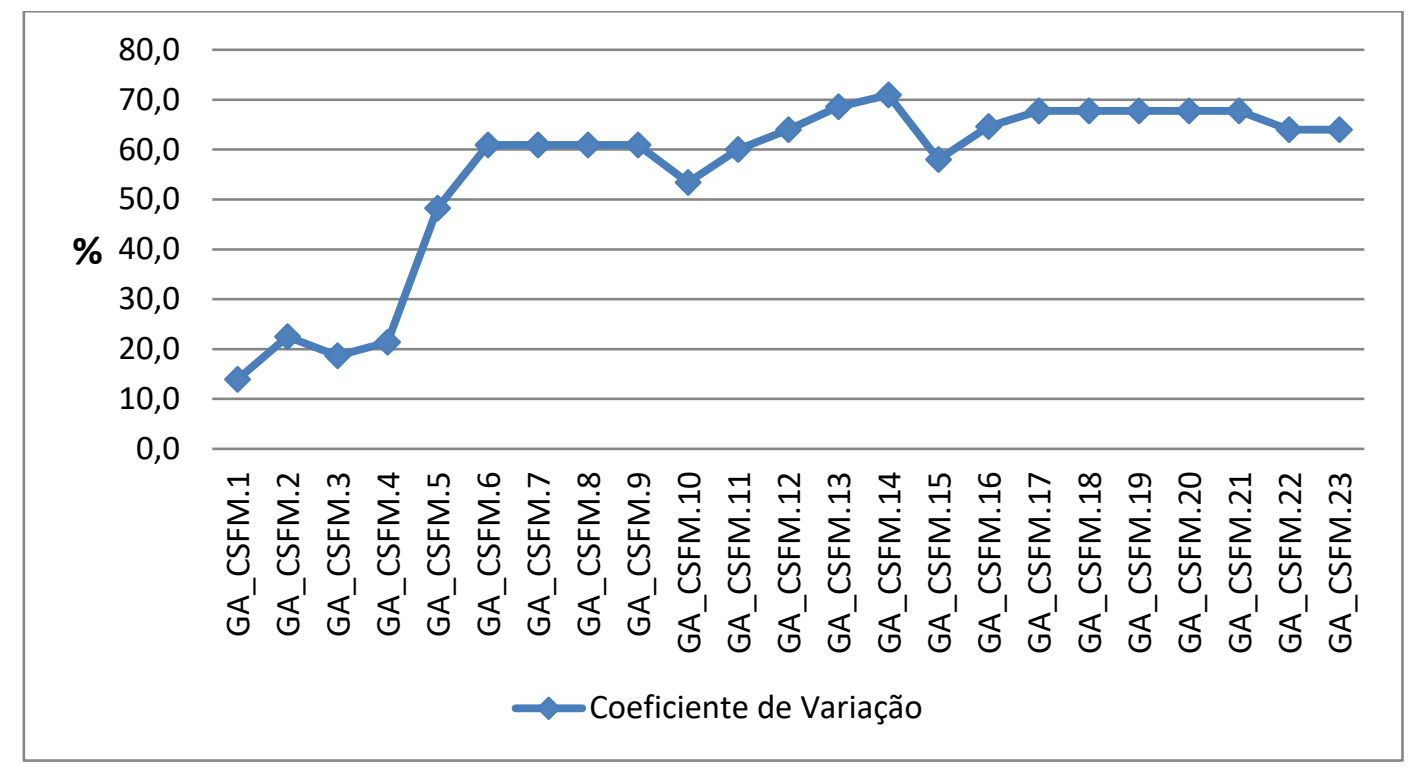

Figura 2: Coeficiente de variação dos critérios de seleção de fornecedores.

No que concerne às variáveis de competitividade para a seleção de fornecedores de cerâmica vermelha, $35,7 \%$ das construtoras sempre adotam como critério o preço do produto (GA_CSFM.1), 21,4\% a facilidade na compra (GA_CSFM.2) e 28,6\% das construtoras sempre optam pela qualidade dos materiais (GA_CSFM.3) e pela confiabilidade do prazo de entrega (GA_CSFM.4). Nesse sentido, essas variáveis apresentaram os menores percentuais referentes ao coeficiente de variação, demonstrando homogeneidade das respostas.

De outro modo disposto, no tocante aos critérios de seleção considerados como ambientais, $21,4 \%$ sempre adotam os fornecedores que possuem as licenças ambientais em dia (GA_CSFM.5). Ainda com uma menor representatividade de 7,1\%, as construtoras sempre selecionam fornecedores que firmam cláusulas contratuais que estabelecem as rotinas operacionais para o controle ambiental (GA_CSFM.10) e possuem um Plano de Gerenciamento de Resíduos Sólidos (GA_CSFM.15). Desse modo, as construtoras responderam, segundo a escala de variação com cerca de 80 a $100 \%$ da cerâmica vermelha fornecida, que sempre são adotados os critérios supracitados para seleção dos fornecedores.

Nota-se, ainda, que as construtoras selecionam fornecedores que possuem a ISO 14001 implementada (GA_CSFM.6), dispõem a ISO 14001 implementada e certificada (GA_CSFM.7), possuem a certificação OHSAS 18001 (GA_CSFM.8), realizam auditorias ambientais periodicamente (GA_CSFM.12), possuem programas de rótulos ambientais (GA_CSFM.22) e listam as substâncias perigosas usadas no processo (GA_CSFM.23). Todas essas variáveis apresentam um percentual 7,1\% das construtoras pesquisadas. Sendo assim, de acordo com a escala de variação, com cerca de 50 a $80 \%$ do material fornecido, quase sempre os critérios mencionados são adotados para seleção dos fornecedores, muito embora apresentem uma dispersão alta e dados heterogêneos.

Diante disso, é notório perceber que as variáveis com maior representatividade são "GA_CSFM.1", preço do produto; "GA_CSFM.2", facilitação da compra com as formas de pagamento; "GA_CSFM.3", qualidade dos materiais; e "GA_CSFM.4", confiabilidade no prazo de entrega. Tais elementos podem ser considerados como variáveis de competitividade dentro da 
CSV, como atestam Souza, Kliemann Neto e Anzanello (2012). Esses autores enfatizam que tais requisitos são fundamentais para competitividade da empresa frente ao mercado, tendo em vista que, na construção civil no RN, houve um aumento relativo à participação das atividades econômicas no valor adicionado de $6,77 \%$.

No que concerne aos critérios ambientais, apenas o parâmetro de seleção de fornecedores de materiais (representado pela variável "GA_CSFM.5"), o qual requer que os fornecedores possuam as licenças ambientais em dia, apresentou melhor significância. De acordo com Zhu, Sarkis e Lai (2007a), este tipo de medida reguladora impulsiona os fornecedores a realizares as práticas de GCSV. Tendo em vista os percentuais apresentados para esta variável e levando em consideração a função da gestão ambiental das organizações, $35,7 \%$ não possuem o setor ambiental, 35,7\% das atribuições dessa função são destinadas a um profissional da empresa, porém não existe o setor, e $21,4 \%$ encontram-se em implantação, confirmando, assim, a baixa expressividade de um critério primordial para a CSV.

Quanto aos critérios que requerem as certificações, como a ISO 14001, esses não tiveram representatividade. Nawrocka, Brorson e Lindhqvist (2009) enfatizam que os sistemas de gestão ambiental são ferramentas fundamentais para a obtenção de resultados positivos no desempenho da CSV. Desse modo, ainda é notório perceber a ausência de práticas mais amigáveis, como pregam Azevedo, Carvalho e Machado (2011), as quais são importantes para o "greening" da cadeia de suprimentos. Sendo assim, estes autores mencionam que as compras amigáveis é a pratica ambiental menos considerada, como é apontada pelos resultados obtidos na variável "GA_CSFM.10". Tais pesquisadores ainda enfatizam que a ISO 14001 é um critério de seleção de fornecedores, porém é pouco utilizada pelas construtoras, como mostra as variáveis "GA_CSFM.6" e "GA_CSFM.7", exigindo que seus fornecedores se certifiquem nesta norma.

Assim, de acordo com Jabbour e Jabbour (2009), é percebido, nesse cenário, que as construtoras não sistematizam a ISO 14001 como critério de seleção de fornecedores, integrando as diversas fases de análises do ciclo de vida nas construções (analisado pela variável "GA_CSFM.21"), pois 35,7 (0\% do material fornecido) "nunca" adotam essa norma para seleção dos fornecedores.

Para tanto, a realidade das construtoras, objeto da pesquisa, enquadram-se no perfil de empresas preventivas, haja vista que não fazem uso, de forma satisfatória, dos critérios de seleção de fornecedores de materiais, o que passaria a aumentar o nível de desempenho da CSV do setor da construção, em um cenário no qual a cadeia de suprimentos pode ser considerada uma prática ambiental proativa, quando sistematizada (Jabbour \& Jabbour, 2009). Apesar disso, empresas automobilísticas chinesas têm melhorado o desempenho ambiental e econômico com práticas de GCSV, a partir de medidas reguladoras mais rígidas, como o licenciamento ambiental (GA_CSFM.5), o mercado com ofertas mais inovadoras e produtos mais ecológicos (Zhu, Sarkis \& Lai, 2007a).

\section{CONSIDERAÇÕES FINAIS}

Diante de tudo o que foi mencionado, a pesquisa se mostrou de fundamental importância para o conhecimento de como as construtoras inserem a variável ambiental da tomada de decisão na seleção de fornecedores de cerâmica vermelha. 
No que diz respeito aos critérios de seleção de fornecedores considerados de competitividade, esses demonstraram ser os mais adotados pelas construtoras, pelo fato de manterem uma relação mais fiel com os fornecedores, como a relacionada ao preço, correspondendo ao percentual de $35,7 \%$ de adoção das construtoras estudadas. Desse modo, o critério ambiental com maior percentual mostrou um valor de $21,4 \%$, exigindo que os fornecedores detenham as licenças ambientais em dia. No tocante a outros critérios ambientais, como a implantação da ISO 14001, a implementação e a certificação são critérios que demostraram, às construtoras, pouca importância para a seleção de fornecedores, haja vista que $35,7 \%$ das empresas não possuem o setor ambiental e $28,6 \%$ delas estavam no mercado há cerca de 05 (cinco) a 10 (dez) anos.

Diante dos resultados, observa-se que as construtoras não detêm uma sistematização referente às práticas ambientais, configurando a CSV, o que passa a comprometer o seu desempenho ambiental, pois, de acordo com a análise do grupo de variáveis "GA_CSFM", as construtoras priorizam os critérios de competitividade. Nesse sentido, as construtoras deveriam interagir junto aos seus fornecedores de cerâmica vermelha, exigindo melhores resultados ambientais, tendo em vista que os consumidores e o governo estabelecem exigências quanto à qualidade dos serviços e dos produtos. Sendo assim, aplicar as práticas da GSC dentro da cadeia produtiva da construção civil de Natal é algo a ultrapassar barreiras, como os custos de investimento em tecnologias ambientais. Assim, faz-se necessária uma gestão que enalteça a reputação das construtoras, objeto de estudo desta pesquisa.

No que concerne às limitações deste estudo, nota-se o baixo percentual de empresas que responderam e enviaram os questionários, o que comprometeu o método da pesquisa adotada, a survey. As lacunas em branco deixadas pelos participantes também foram fatores que afetaram o perfil socioeconômico das empresas e dos respondentes. Nesse sentido, acredita-se que a grande quantidade de variáveis tornou-se, no ato da resposta, cansativo para os informantes e, consequentemente, por ser o último grupo de variáveis e pela complexidade e necessidade de dados mais detalhados, as variáveis que buscavam analisar os critérios estabelecidos pelas construtoras nos processos de seleção de fornecedores de materiais não foram respondidas por $50 \%$ das empresas participantes.

Por fim, conhecer mais detalhadamente a CSV no setor da construção civil, partindo de uma avaliação dos impactos ambientais dos fornecedores das empresas foco (construtoras) deste setor, seria um estudo pertinente para a comunidade científica e para a iniciativa privada, tendo em vista que, por meio dos resultados obtidos com estudos futuros, seriam levantadas as oportunidades de melhoria a partir de uma gestão ambiental proativa, como a GCSV.

\section{REFERÊNCIAS}

Azevedo, S.; Carvalho, H. \& Machado, V. C. (2011) The influence of green practices on supply chain performance: A case study approach. Transportation Research Part E: Logistics and Transportation Review, v. 47, p. 850-871, 2011.

Bozarth, C. C. \& Handfield, R. B. (2008) Introduction to operations and supply chain management. New Jersey: Pearson Education Inc. 
Brito, P. B. \& Berardi, P. C. (2010) Vantagem competitiva na gestão sustentável da cadeia de suprimentos: um meta estudo. Revista de Administração de Empresas, São Paulo, v. 50, n. 2, p. 155-169.

Ehrlich, P. J. (1996). Modelos quantitativos de apoio às decisões. Revista de Administração de Empresas, 36(1), 33-41. doi: 10.1590/S0034-75901996000100006

Freitas, H., Oliveira, M., Saccol, A. Z., Moscarola, J. (2000) O método de pesquisa survey. Revista de Administração, São Paulo, v. 35, n. 3, p. 105-112.

Günther, H. (2003). Como elaborar um questionário. (Série: planejamento de pesquisa nas Ciências Sociais, n. 1). Brasília, DF: UnB, Laboratório de Psicologia Ambiental, p.1-15.

Günther, H. (2006). Pesquisa Qualitativa Versus Pesquisa Quantitativa: Esta É a Questão? Psicologia: Teoria e Pesquisa, v. 22, n. 2, p.201-210.

Hervani, A. A., Helms, M. M. \& Sarkis, J. (2005). Performance measurement for green supply chain management. Benchmarking: An International Journal, v. 12, n. 4, p. 330-353.

Jabbour, A. B. L. S. \& Jabbour, C. J. C. (2009). Are supplier selection criteria going green? Case studies of companies in Brazil. Industrial Management and Data System, v. 4, p. 477-495.

Lambert, D. M. (2008). Supply chain management: processes, partnerships, performance. Sarasota: SCM Institute.

Nawrocka, D., Brorson, T. \& Lindhqvist, T. (2009). ISO 14001 in environmental supply chain practices. Journal of Cleaner Production, v. 17, p. 1435-1443.

Seuring, S. \& Muller, M. (2008). From a literature review to a conceptual framework for sustainable supply chain management. Journal of Cleaner Production, v. 16, p. 1699-1710.

Souza, D. V., Klienmann Neto, F. J. \& Anzanello, M. J. (2012). Avaliação de desempenho da cadeia de suprimentos balizada por critérios de competitividade empresarial. Revista Produção Online, v.12, n. 3, p. 756-778.

Zhu, Q. \& Sarkis, J. (2004). Relationships between operational practices and performance among early adopters of green supply chain management practices in Chinese manufacturing enterprises. Journal of Operations Management, v. 22, p. 265-289.

Zhu, Q., Sarkis, J. \& Lai, K. (2007a). Green supply chain management: pressures, practices and performance within the Chinese automobile industry. Journal of Cleaner Production, v. 15, p. 1041-1052.

. (2007b). Initiatives and outcomes of green supply chain management implementation by Chinese manufacturers. Journal of Environmental Management, v. 85, p. 179-189. 